\title{
Experimental demonstration of the relationship between the second- and third-order polarizabilities of conjugated donor-acceptor molecules
}

\author{
Grant Bourhill, ${ }^{\text {a Lap-Tak Cheng, }}{ }^{\text {b }}$ Christopher B. Gorman, ${ }^{\text {a,c }}$ Ging Lee, ${ }^{c}$ Seth R. Marder, ${ }^{\text {a,c }}$ \\ Joseph W. Perry, ${ }^{\text {a,c }}$ Matthew J. Perry ${ }^{c}$ and Bruce G. Tiemann ${ }^{\mathrm{a}, \mathrm{c}}$
}

\begin{abstract}
a: Jet Propulsion Laboratory, 67-201, California Institute of Technology, Pasadena, California 91109.
b: Central Research and Development, Science and Engineering Laboratories, E. I. Du Pont de Nemours \& Co. (Inc.), Experimental Station, P. O. Box 80356, Wilmington, Delaware 19880-0356.

c: The Beckman Institute, 139-74, California Institute of Technology, Pasadena, California 91125.
\end{abstract}

\begin{abstract}
The dependence of the second- and third-order polarizabilities ( $\beta$ and $\gamma$ ) on ground-state polarization was measured for a series of donor-acceptor polyenes using electric field induced second harmonic generation and third harmonic generation, respectively. The changes in ground-state polarization, associated with the donor/acceptor strength or solvent polarity, were probed by $\mathrm{x}$-ray crystallography, ${ }^{1} \mathrm{H}-\mathrm{NMR}$, electronic absorption and Raman spectroscopies. The observed behavior of $\beta$ and $\gamma$ as a function of ground-state polarization agrees well with theoretical predictions. In particular, positive and negative peaks, as well as sign changes, were observed for both $\beta$ and $\gamma$. The dependences for $\beta$ and $\gamma$ are consistent with a derivative relationship between them. In addition, the third-order polarizability of a series of molecules possessing zero bond length alternation was found to be negative, in agreement with predictions based on the relationship between the polarizabilities and ground-state geometry.
\end{abstract}

\section{INTRODUCTION}

Recent studies 1,2 have suggested that optimizing the polarizabilities of donor-acceptor polyenes requires a specific degree of mixing between neutral and charge-separated resonance structures. The degree of mixing is related to the donor/acceptor strength and can be correlated with the ground-state polarization (i.e. the dipole moment) and a molecular parameter, bond length alternation (BLA), defined as the difference between the average single and double carbon-carbon bond lengths in the polymethine chain. Figure 1 illustrates how chemical modifications can systematically tune the bond length alternation by varying the degree of mixing between neutral and charge-separated structures. For unsubstituted polyenes, or chromophores with weak donors/acceptors, $\mathbf{A}$, the ground-state structure resembles that of the neutral resonance form resulting in large positive BLA. ${ }^{3}$ Increasing the mixing between the two limiting resonance forms by utilizing the stronger dicyanovinyl acceptor, B, results in increased ground-state polarization and smaller BLA. ${ }^{3}$ Complete mixing of the limiting resonance forms results in essentially zero BLA, as for symmetrical cyanines, C. ${ }^{4}$ In neutral donor/acceptor systems, increasing the donor and/or acceptor strength further, $\mathrm{D}$, results in the charge-separated resonance form being the dominant contributor to the ground state, resulting in negative BLA. 5

For a particular chromophore, the degree of BLA can be fine-tuned by utilizing solvents of varying polarity since the degree of mixing between neutral and charge-separated resonance structures is known to be sensitive to this perturbation. ${ }^{3,6,7}$ Since polar solvents can stabilize the charge-separated structure to a larger extent than nonpolar solvents, the ground-state polarization of a donor-acceptor molecule can thus be increased by utilizing increasingly polar solvents, since this increases the contribution of the charge-separated form to the ground-state geometry. 


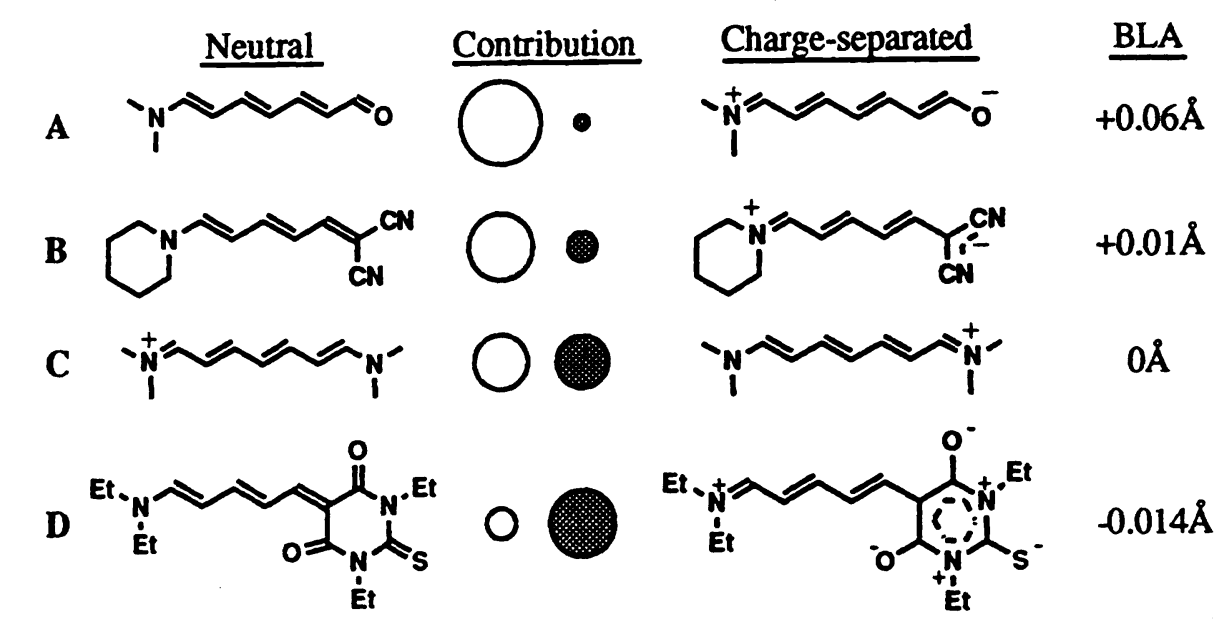

Figure 1. Contribution of neutral and charge-separated resonance forms, given qualitatively by the area of the open and shaded circles respectively, to the ground state. BLA values, tuned by varying donor/acceptor strengths, were determined from $x$-ray diffraction. ${ }^{3-5} \mathrm{Et} \equiv \mathrm{C}_{2} \mathrm{H}_{5}$.

Quantum mechanical calculations have been used to predict the dependence of the polarizability of donor-acceptor molecules on bond length alternation. 2,8 The predicted correlation of the first-, second- and third-order polarizability ( $\alpha, \beta$ and $\gamma$ respectively) with bond length alternation is depicted in Figure 2 where it can be seen that: (i) the polarizabilities can be optimized as a function of BLA; (ii) relationships between the various order polarizabilities exist, which are consistent with the $\mathrm{n}^{\text {th }}$-order polarizability being related to the derivative of the $(n-1)^{\text {th }}$-order polarizability. In this paper, the polarizabilities of a series of donor-acceptor polyenes exhibiting a wide range of ground-state polarization were probed to test the structure-property relationships depicted in Figure 2.

\section{THEORETICAL DEPENDENCE OF THE POLARIZABILITIES ON BLA}

A detailed explanation of the predicted dependence of the polarizabilities on BLA is presented elsewhere, ${ }^{8}$ but briefly it can be understood by consideration of the behavior of the terms in simplified sum-over-states expressions for $\alpha, \beta$ and $\gamma$, as a function of BLA.

$$
\begin{gathered}
\alpha \propto \frac{\left(\mu_{\mathrm{ge}}\right)^{2}}{\mathrm{E}_{\mathrm{ge}}} \\
\beta \propto \frac{\left(\mu_{\mathrm{ge}}\right)^{2}\left(\mu_{\mathrm{ee}}-\mu_{\mathrm{gg}}\right)}{\left(\mathrm{E}_{\mathrm{ge}}\right)^{2}} \propto \frac{\alpha\left(\mu_{\mathrm{ee}}-\mu_{\mathrm{gg}}\right)}{\mathrm{E}_{\mathrm{ge}}} \\
\gamma \propto-\frac{\left(\mu_{\mathrm{ge}}\right)^{4}}{\left(\mathrm{E}_{\mathrm{ge}}\right)^{3}}+\frac{\left(\mu_{\mathrm{ge}}\right)^{2}\left(\mu_{\mathrm{ee}}\right)^{2}}{\left(\mathrm{E}_{\mathrm{ge}}\right)^{2} \mathrm{E}_{\mathrm{ge}}}+\frac{\left(\mu_{\mathrm{ge}}\right)^{2}\left(\mu_{\mathrm{ee}}-\mu_{\mathrm{gg}}\right)^{2}}{\left(\mathrm{E}_{\mathrm{ge}}\right)^{3}} \\
\mathrm{P} 1
\end{gathered}
$$

where $\mu$ and $\mathrm{E}$ are the dipole matrix element and transition energy, respectively, between the subscripted states. The subscripts $\mathrm{g}$, e and $\mathrm{e}^{\prime}$ label the ground, first excited and upper excited states respectively. 


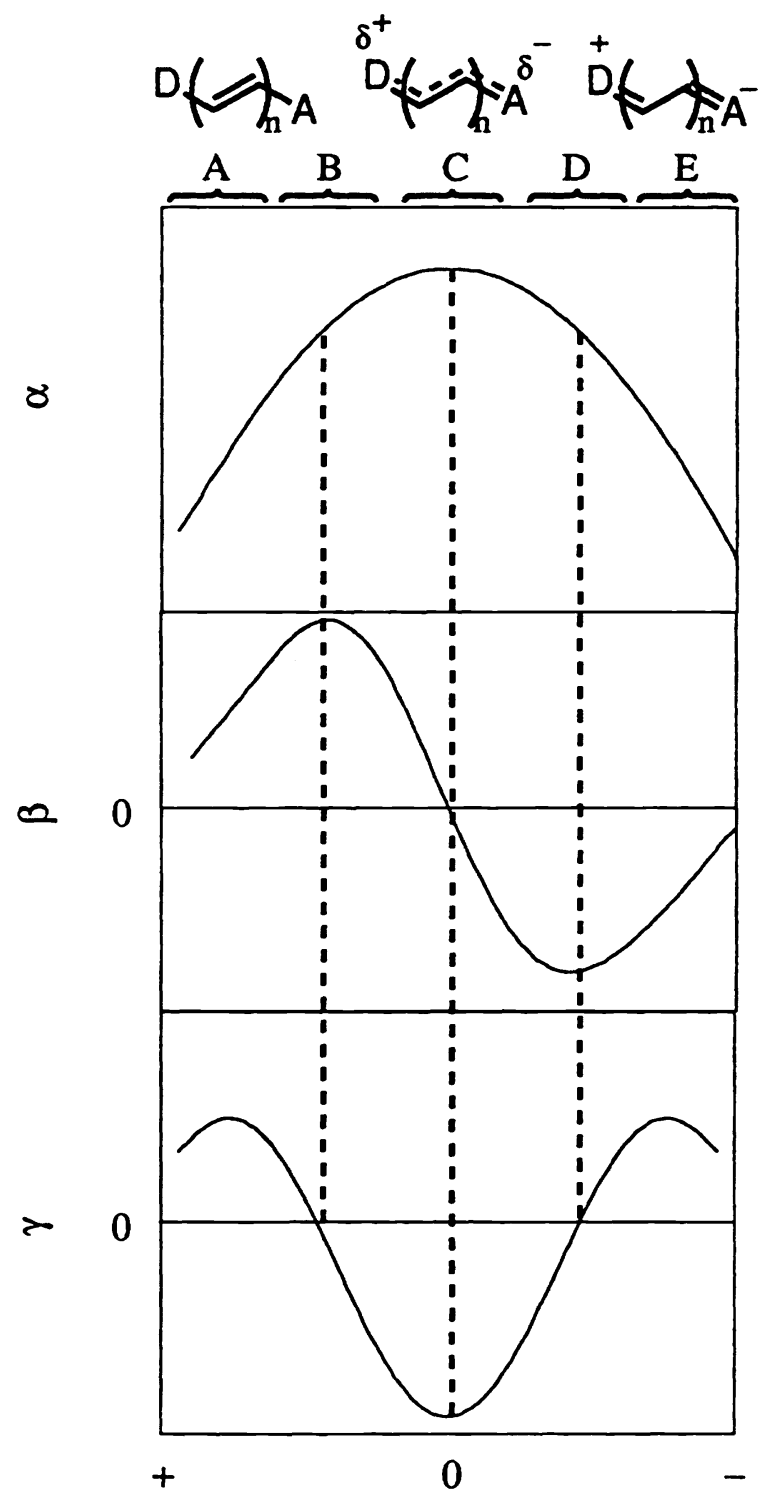

\section{Bond Length Alternation}

Figure 2. Schematic illustration of the predicted dependence of $\alpha, \beta$ and $\gamma$ on bond length alternation. The ground-state polarization increases from $\mathrm{A}$ to $\mathrm{E}$.

The first-order polarizability, $\alpha$, is dependent on two terms, $\left(1 / \mathrm{E}_{\mathrm{ge}}\right)$ and $\mu_{\mathrm{ge}}$, which both peak ${ }^{1}$ at the cyanine-limit of zero bond length alternation (Figure 3), explaining the dependence of $\alpha$ on BLA in Figure 2 . The two-level model ${ }^{9}$ for $\beta$ is similar to an $\alpha$-term scaled by a factor of $\left(\mu_{\mathrm{ee}}-\mu_{\mathrm{gg}}\right) / \mathrm{E}_{\mathrm{ge}}$. The difference in dipole moment between the ground and excited state, $\left(\mu_{\mathrm{ee}}{ }^{-}\right.$ $\mu_{\mathrm{gg}}$ ), exhibits positive and negative peaks at BLA values corresponding to regions A and E respectively (Figure 3). 1,2,8,10 Thus $\beta$, as a function of increasing ground-state polarization, starts positive, increases, peaks in a positive sense, decreases, crosses through zero at the limit of zero BLA, becomes large and negative, peaks in a negative sense and eventually decreases in magnitude (Figure 3). $1,2,8,10 \beta$ displays positive and negative peaks closer to the zero BLA limit (i.e. regions B and D, respectively) than the $\left(\mu_{\mathrm{ee}}-\mu_{\mathrm{gg}}\right)$ peaks (Figure 3$)$ since $\left(1 / \mathrm{E}_{\mathrm{ge}}\right)$ and $\mu_{\mathrm{ge}}$ peak at the zero BLA limit. 


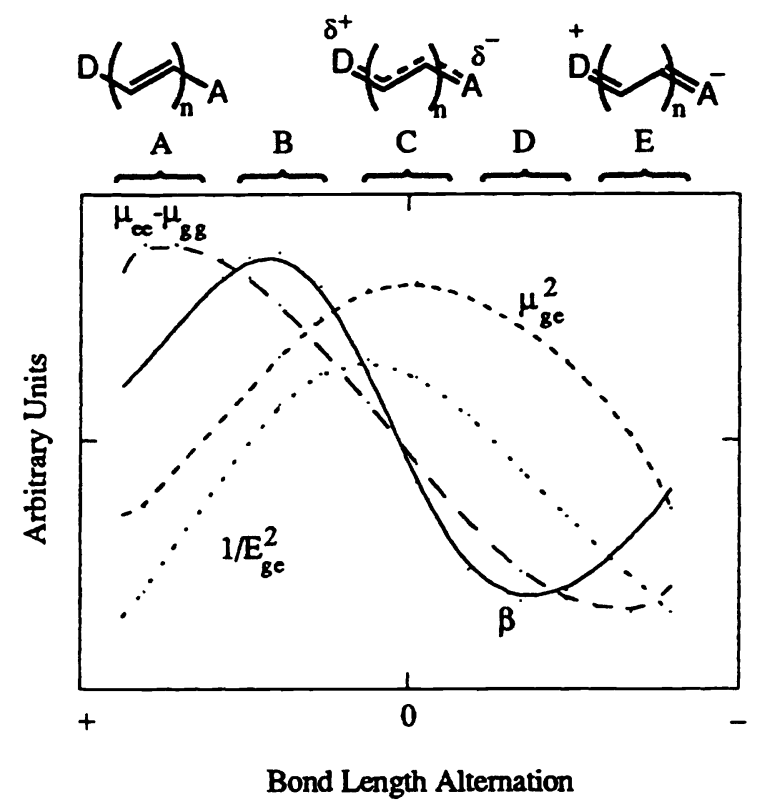

Figure 3. Dependence of $\left(\mu_{\mathrm{ge}}\right)^{2},\left(1 / \mathrm{E}_{\mathrm{ge}}\right)^{2},\left(\mu_{\mathrm{ee}}-\mu_{\mathrm{gg}}\right)$ and $\beta$ on the ground-state structure. The ground-state polarization increases from $A$ to $\mathrm{E}$. The point where $\left(\mu_{\mathrm{ee}}-\mu_{\mathrm{gg}}\right)$ and $\beta$ are zero corresponds to the bond-equivalent cyanine-limit of zero bond length alternation. The curves were calculated ${ }^{8}$ for $\mathrm{Me}_{2} \mathrm{~N}-(\mathrm{CH}=\mathrm{CH})_{4}-\mathrm{CHO}$, geometry-optimized in the presence of a static electric field (used to modify the geometry from the highly bond-alternate polyene-limit (region A), through the bond-equivalent cyanine-limit (region C) to the bond-alternate charge-separated polyene-limit (region E)), at the semiempirical INDO level using a sum-over-states approach in combination with an INDO-configuration interaction technique that includes full single and limited double configuration excitations.

The three-level model ${ }^{11-13}$ for the third-order polarizability, $\gamma$, consists of a negative, $\alpha^{2}$-like term, N1, a two-photon term, $\mathrm{P} 1$, and a $\beta$-like term, $\mathrm{P2}$. For chromophores with large positive BLA, such as unsubstituted polyenes, the P2 term is negligible due to small $\left(\mu_{\mathrm{ee}}-\mu_{\mathrm{gg}}\right)$ and the P1 term dominates N1 resulting in positive $\gamma$ (Figure 2). ${ }^{7,11}$ By increasing the ground-state polarization and thus decreasing BLA, the P2 term, like $\beta$ (Figures 2 and 3), starts to increase and hence $\gamma$ increases. However, increasing the ground-state polarization toward the cyanine-limit (region $\mathrm{C}$ ) also increases $\mathbf{I N} 11$ and therefore a positive peak in the $\gamma$ curve is evident at larger magnitudes of BLA than the peak in the $\beta$-like P2 term. Upon further polarization, P2 peaks in a positive sense and starts to decrease and $\mid \mathrm{N} 1 \mathrm{l}$ continues to increase and thus $\gamma$ decreases in magnitude until $\mathbb{N} 1 \mid=P 1+$ $\mathrm{P} 2$ and $\gamma$ is zero. 7,8 At the bond-equivalent cyanine-limit (region C), the P2 term is zero since $\left(\mu_{\mathrm{ee}} \mu_{\mathrm{gg}}\right.$ ) is zero. The $\gamma$ curve exhibits a negative peak at this point since both $\mathbb{N} 11$ and P1 peak but $\mathbb{N} 11>\mathbb{I P 1 I . 7 , 8}$ To a first approximation, the behavior of $\gamma$ in the region of negative BLA mirrors that in the positive BLA region (Figure 2).

The observation that $\alpha, \beta$ and $\gamma$ appear to be related by a derivative-like relationship can be understood by considering a Taylor series expansion of the dipole moment,

$$
\mu=\mu_{0}+\alpha E+\frac{1}{2} \beta E^{2}+\frac{1}{6} \gamma E^{3}+\ldots
$$

where

$$
\alpha=\left.\frac{\partial \mu}{\partial E}\right|_{0} \quad \beta=\left.\frac{\partial^{2} \mu}{\partial E^{2}}\right|_{0} \quad \gamma=\left.\frac{\partial^{3} \mu}{\partial E^{3}}\right|_{0}
$$


so that

$$
\beta=\left.\frac{\partial \alpha}{\partial \mathrm{E}}\right|_{0} \quad \gamma=\left.\frac{\partial \beta}{\partial \mathrm{E}}\right|_{0}
$$

Now if we consider a molecule to be polarized by a strong field, $F$, that can modify the degree of bond length alternation associated with the molecule, the dipole moment will depend on $\mathrm{F}$ in some characteristic way,

$$
\mu=\mu(\mathrm{F})
$$

for which a single Taylor series with constant coefficients is not a sufficient description. Qualitatively, we would expect, and AM-1, ${ }^{2}$ INDO ${ }^{8}$ and valence bond calculations ${ }^{14}$ show: (i) that for weak fields, such that the BLA is polyene-like, $\mu$ will be small and weakly dependent on field; (ii) that when the field is large, such that the structure is close to the bond-altemate chargeseparated polyene-limit, $\mu$ will be large and weakly dependent on field; (iii) that for moderate fields, $\mu$ will be intermediate and will vary most rapidly with field, i.e. the rate of change of the BLA with field is largest at the cyanine-limit.

At any particular value of $F$, say $F$, we can expand $\mu$ in a Taylor series, and thus

$$
\alpha=\left.\frac{\partial \mu}{\partial F_{F^{\prime}}}\right|_{F^{\prime}} \quad \beta=\left.\frac{\partial \alpha}{\partial F}\right|_{F^{\prime}}
$$

Therefore the functions $\alpha(F), \beta(F)$ and $\gamma(F)$ will be given by

$$
\alpha(F)=\frac{\partial \mu(F)}{\partial F} \quad \beta(F)=\frac{\partial \alpha(F)}{\partial F} \quad \gamma(F)=\frac{\partial \beta(F)}{\partial F}
$$

leading to derivative relationships between a given polarizability and the lower-order polarizability. Keeping in mind that the ground-state polarization, and hence BLA, is a function of the field strength, i.e. BLA = BLA(F), that if we want to map the polarizabilities as a function of BLA, that the change of variables is nonlinear, i.e.

$$
\alpha=\frac{\partial \mu}{\partial \mathrm{F}} \frac{\partial \mathrm{F}}{\partial \mathrm{BLA}}
$$

since BLA is a nonlinear function of F. This leads to a change of the shape of the $\alpha, \beta$ and $\gamma$ curves versus BLA compared to those versus F. Nonetheless, the relation of the peaks and zero crossings of the different polarizabilities, e.g. the peak of $\alpha$ and the zero crossing of $\beta$, are preserved and we will use these special points to test experimentally the validity of these relationships.

\section{MODEL COMPOUNDS AND DEGREES OF BLA}

The molecules (Figure 4) studied to test the predicted structure-property relationships were chosen since strong evidence exists that they approximately cover the BLA range A-E in Figure 2.10 The bond length alternation values were coarsely changed by utilizing donors and acceptors of varying strength and fine-tuned using solvent polarity. Bond length alternations, determined from $x$-ray crystallography, of unsubstituted polyenes and 3, containing the weak formyl acceptor, have been reported previously 3,15 and allow an assignment of 1-3 to region A. This assignment is consistent with the positive solvatochromism displayed by 3 in all solvents used (Table I). 
1<smiles>CC=CC=CC=CC=CC</smiles>

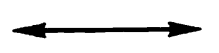<smiles>C[C+]C=CC=CC=CCC</smiles>

Positive

BLA

2<smiles>CC=CC=CC=CC=CC</smiles>

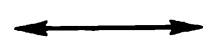<smiles>C[C+]C=CC=CC=CCC</smiles>

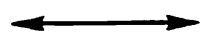

3<smiles>CN(C)/C=C/C=C/C=C/C=O</smiles>

4

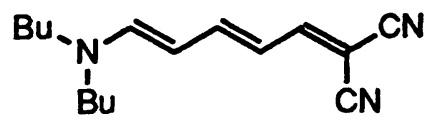

5<smiles>CCN(/C=C/C=C/C=C1C(=O)N(CC)C(=O)N(CC)C1=O)CC</smiles>

6<smiles>C=CN(C=CC=CC=C1C(=O)N(CC)C(=S)N(CC)C1=O)CC</smiles><smiles>C1CCCCC1</smiles><smiles>C[N+](C)=CC=CC=CC=C[O-]</smiles>

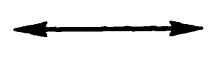<smiles>C[N+](=CC=CC=CC(C)(C)C)CBr</smiles><smiles>CC[N+](=CC=CC=Cc1c([O-])[n+](CC)c([O-])n1[O-])CC</smiles><smiles>CC[N+](=CC=CC=Cc1c(O)[n+]([O-])c([S-])[n+](CC)c1[O-])CC</smiles>

7<smiles></smiles>

8<smiles>CCN1C(=O)C(=CC=C2C=CN(CCC(C)C)C=C2)C(=O)N(CC)C1=S</smiles>

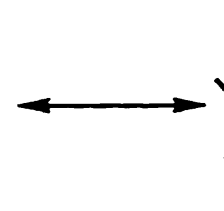<smiles>CCCC(C)C</smiles><smiles>C[n+]1ccccc1</smiles><smiles>C/C=C/c1c([O-])[n+](CC)c([S-])[n+](CC)c1[O-]</smiles>

Negative

BLA

Figure 4. Canonical resonance structures for the donor-acceptor polyenes investigated. Electron donor/acceptor strength increases from 3 to 8. $\mathrm{Et} \equiv \mathrm{C}_{2} \mathrm{H}_{5}$ and $\mathrm{Bu} \equiv \mathrm{n}-\mathrm{C}_{4} \mathrm{H}_{9}$.

Compound 4 with the stronger dicyanovinyl acceptor possesses a bond length alternation of $+0.015 \AA$ in the solid state, assigning this molecule to region B. Interestingly, this small BLA value suggests that in polar solvents the ground-state geometry is cyanine-like whereas in nonpolar solvents, due to a decreased contribution of the charge-separated state, the groundstate structure is more similar to an unsubstituted polyene. These solvent-dependent geometry changes on a related molecule (B, Figure 1) were probed by non-resonant Raman spectroscopy (Figure 5). ${ }^{3}$ The Raman spectrum of the unsubstituted polyene, (Figure 5a), exhibits two main bands, those due to the $C-C\left(1150 \mathrm{~cm}^{-1}\right)$ and $C=C$ modes $\left(1600 \mathrm{~cm}^{-1}\right)$. The Raman spectrum of the bond-equivalent cyanine, (Figure 5d), exhibits increased activity at intermediate frequencies consistent with the loss of discrete $\mathrm{C}-\mathrm{C}$ and $\mathrm{C}=\mathrm{C}$ character. As predicted, the Raman spectrum of the dicyanovinyl moiety in nonpolar solvents $\left(\mathrm{CHCl}_{3}, \mathrm{Figure}\right.$ $5 b)$ is qualitatively similar to that of the polyene whereas the spectrum in polar solvents $\left(\mathrm{CH}_{3} \mathrm{CN}\right.$, Figure $\left.5 \mathrm{c}\right)$ is qualitatively similar to that of the cyanine. The positive solvatochromism displayed by 4 in all solvents used (Table I) is consistent with the structural assignment of region $B$. 

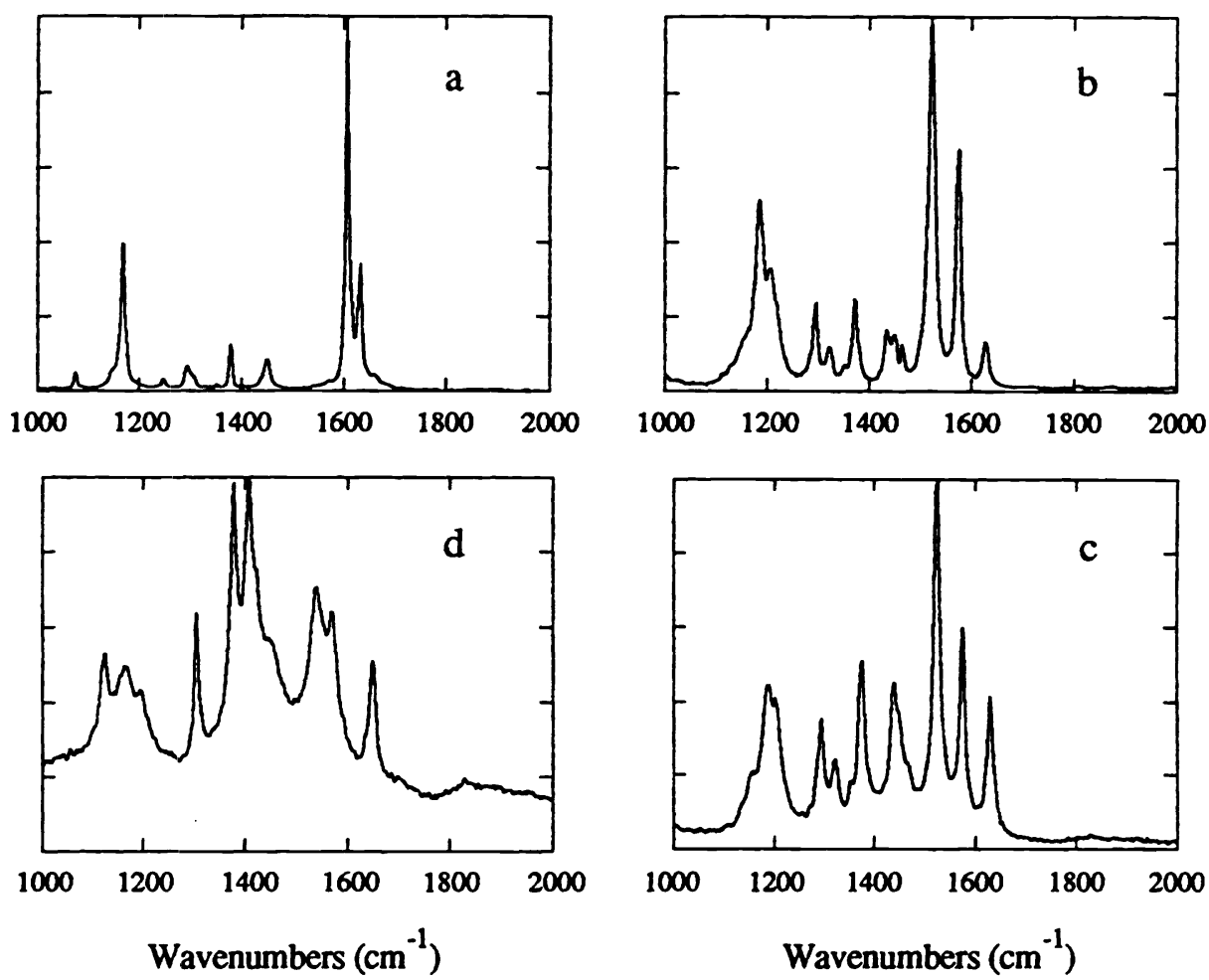

Figure 5. Raman spectra for (a) The polyene decatetraene (1, Figure 4) in the solid state; (b) B (Figure 1) in $\mathrm{CHCl}_{3}$; (c) $\mathrm{B}$ (Figure 1) in $\mathrm{CH}_{3} \mathrm{CN}$; (d) A cyanine (C, Figure 1), in $\mathrm{CH}_{3} \mathrm{CN}^{3}$ The counterion for the cyanine was perchlorate.

The ground-state polarization can be increased (bond length alternation decreased) further by utilizing the barbituric and thiobarbituric acid acceptors, 5 and 6 respectively. In fact, BLA for 6 in the solid state is $-0.014 \AA$ (Figure 1), which indicates that the ground-state geometry is biased slightly towards the charge-separated canonical resonance form. Both 5 and 6 display positive solvatochromism in nonpolar solvents, whereas negative solvatochromism is displayed for 5 in highly polar solvents and 6 in moderate polarity solvents, consistent with 5 being in region B/C and 6 in region $C$.

Molecules 7 and 8 display negative solvatochromism in all solvents used. Furthermore, the large ${ }^{1} \mathrm{H}-{ }^{1} \mathrm{H}$ coupling constants (Figure 6) across the central carbon-carbon bond are consistent with a trans double bond (as depicted in the zwitterionic form in Figure 4, right) assigning 7 and 8 to regions $C / D$ and $D / E$ respectively. These coupling constants increase with increasing solvent polarity, consistent with an evolution of the ground-state geometry towards a more charge-separated structure.

The product of the ground-state dipole moment, $\mu$, and the second-order polarizability, $\beta$, was determined by solventdependent, electric field induced second harmonic generation (EFISH) measurements at $1907 \mathrm{~nm}$ using apparatus and methodology described elsewhere. ${ }^{17}$ The third-order polarizability, $\gamma$, was determined by solvent-dependent third harmonic generation (THG) measurements at $1907 \mathrm{~nm}$. A description of the experimental apparatus and data reduction scheme has been reported previously. 18 


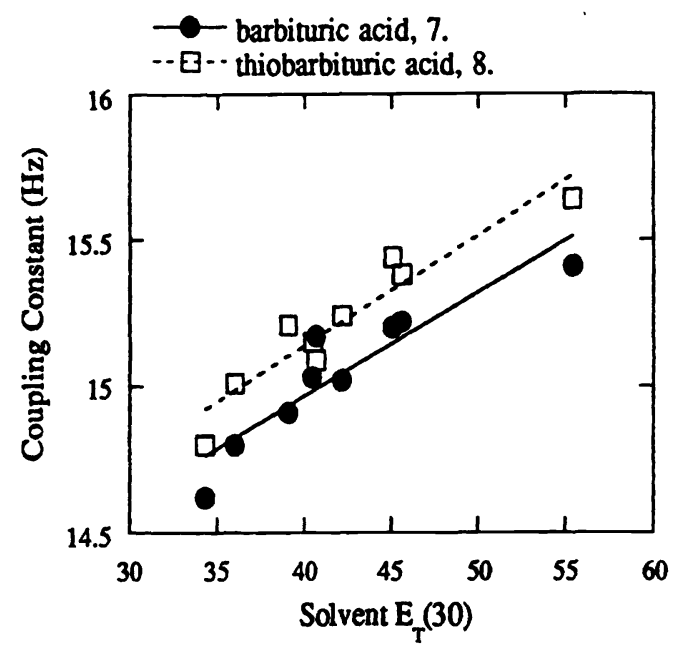

Figure 6. The ${ }^{1} \mathrm{H}-{ }^{1} \mathrm{H}$ coupling constants for 7 and 8 as a function of solvent polarity. ${ }^{16}$ The trend of increasing coupling constant with increasing solvent polarity is indicative of an evolution towards a more charge-separated ground state (Figure 4, right).

\section{RESULTS AND DISCUSSION}

The solvent-dependent polarizabilities for the molecules in Figure 4 are presented in Table I. Molecules 1 and 2 display positive $\gamma$ values consistent with those expected given the large positive bond length alternation values from previous structure determinations. ${ }^{15}$ The increase of $\gamma$ with increasing conjugation length has been noted by other authors. ${ }^{19}$ Molecule 3, with the weak formyl acceptor, displays an increasing $\mu \cdot \beta$ product and a positive peak in $\gamma$ with increasing solvent polarity. This behavior is consistent with that expected for the structural assignment above of 3 in region A (Figure 2). The increase in $\gamma$ for the donor-acceptor polyene, 3, over that of the symmetrical unsubstituted polyenes 1 and 2 has been noted previously ${ }^{11}$ and is due to the lowering of symmetry with the result that the $P 2$ term (equation 3 ) contributes positively to $\gamma$.

Compound 4 with the stronger dicyanovinyl moiety displays a positive peak in $\mu \cdot \beta$ and a sign change in $\gamma$. In fact, the $\mu \cdot \beta$ peak and the zero crossing of $\gamma$ occur simultaneously, consistent with the derivative-like relationship predicted in Figure 2 and with the structural assignment of 4 in region $B$.

Molecules 5 and 6 display decreasing $\mu \cdot \beta$ products with increasing solvent polarity. For 5 in the most polar solvent and for 6 in moderate polarity solvents, $\mu \cdot \beta$ changes sign, consistent with the ground-state geometry being tuned through the cyaninelimit of zero bond length alternation. The $\gamma$ values for 5 become large and negative with increasing solvent polarity with some evidence that $\gamma$ peaks in a negative sense in the most polar solvents. Molecule 6 displays a clear negative $\gamma$ peak in moderate polarity solvents. The zero crossing of the $\mu \cdot \beta$ curve for both 5 and 6 coincides with the negative $\gamma$ peak, consistent with the proposed structure-property relationships. Additionally, to the extent to which $\alpha$ can be represented by $\left(1 / \mathrm{E}_{\mathrm{ge}}\right), \alpha$ is maximized at the zero crossing of $\mu \cdot \beta$, again consistent with the predicted trend (Figure 2).

By increasing the ground-state polarization further, as with molecules $\mathbf{7}$ and $\mathbf{8}$, there is some evidence that the degree of mixing required to maximize $\mu \cdot \beta$ in a negative sense has been achieved. ${ }^{10}$ A zero-crossing in $\gamma$ for 7 in nonpolar solvents is observed as well as evidence of $\gamma$ approaching a positive peak for 8 in the most polar solvents. The zero crossing of $\gamma$ however does not coincide with the negative peak in $\mu \cdot \beta$. This may be explained by the large ground-state dipole moment shifting the peak in $\mu \cdot \beta$ to lie closer to the $\gamma$ peak than would the $\beta$ peak. 
Table I. Maximum absorption wavelength ( $\lambda$ max, units of $\mathrm{nm}$ ), solvent-dependent $\mu \beta$ (units of $10^{-48}$ esu) and $\gamma$ (units of $10^{-36}$ esu) for 1-8. The estimated precision in $\mu \cdot \beta$ and $\gamma$ is $\pm 15 \%$. The polarity of the solvents increase (the contribution of the charge-separated structure to the ground-state geometry increases) from left to right. Normalized $\mathrm{E}_{\mathrm{T}}(30)$ values ${ }^{16}$ of the solvents are presented within parentheses. Insolubility precluded the determination of the polarizabilities in certain solvents. The $\mu \cdot \beta$ values were determined assuming that the contribution to the EFISH signal from the third-order polarizability, $\gamma$, is negligible. This leads to an error in the $\mu \cdot \beta$ values of $<15 \%$, except when the magnitude of the $\mu \cdot \beta$ values is $<70 \times 10^{-48}$ esu where the error can approach $70 \%$. However, no change in the $\mu \cdot \beta$ trends, from those reported below, is observed when the $\gamma$ contribution is included.

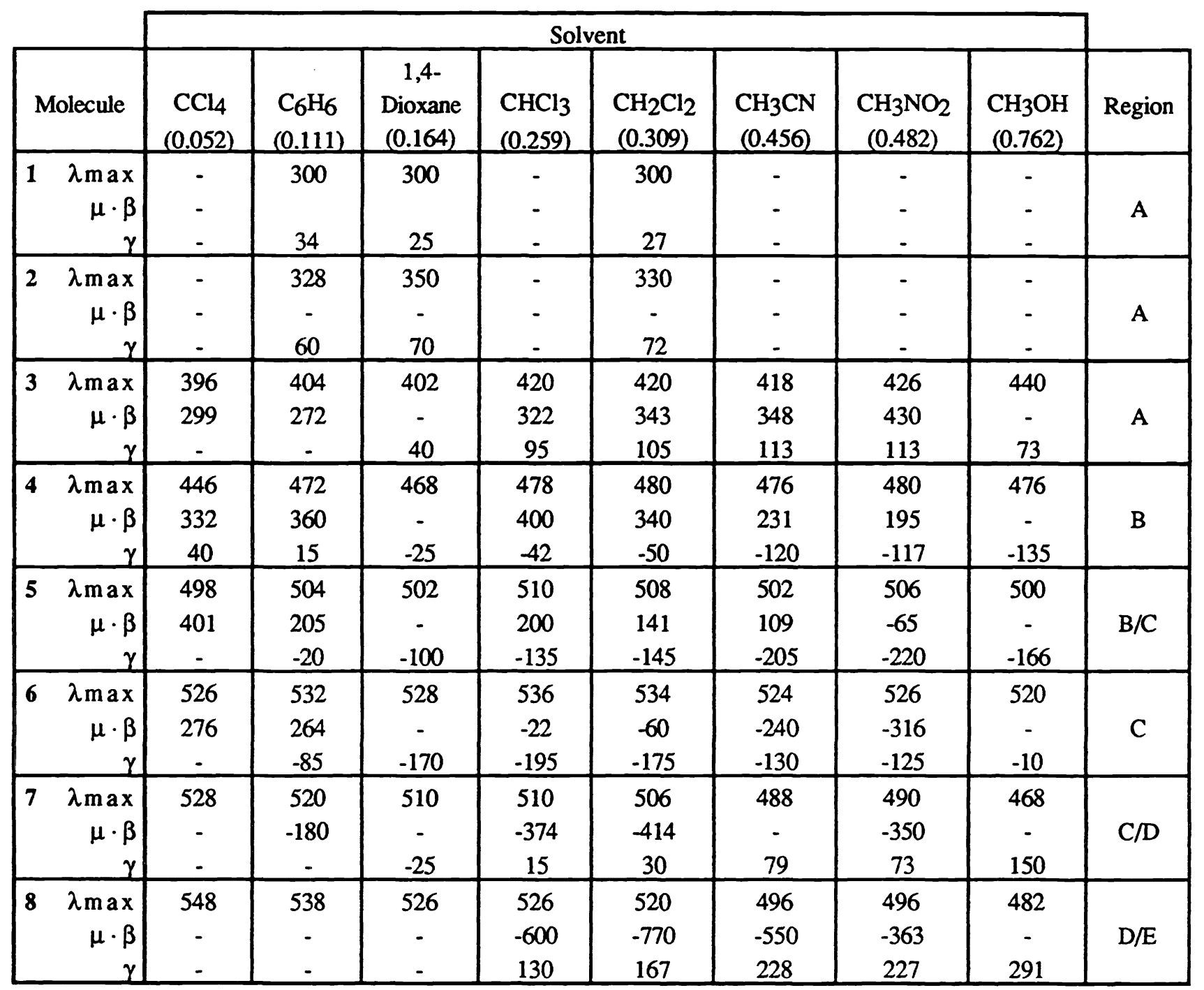

The structure-property relationships proposed in Figure 2 can be further tested by examining the third-order polarizability of molecules possessing zero bond length alternation, for example cyanines and oxonols. The results for a series of these chromophores are presented in Table II where it can be seen that, with the exception of the shortest chromophores, the $\gamma$ values are negative as predicted. 
Table II. $\gamma$ values (units of $10^{-36} \mathrm{esu}$ ) and maximum absorption wavelengths $\left(\lambda_{\max }\right.$, units of $\mathrm{nm}$ ) for molecules possessing zero bond length alternation. The estimated precision in $\gamma$ is $\pm 15 \%$, except in the case where the magnitude of $\gamma<3 \times 10^{-36}$ esu when the estimated error in $\gamma$ is $\pm 5 \times 10^{-36}$ esu.

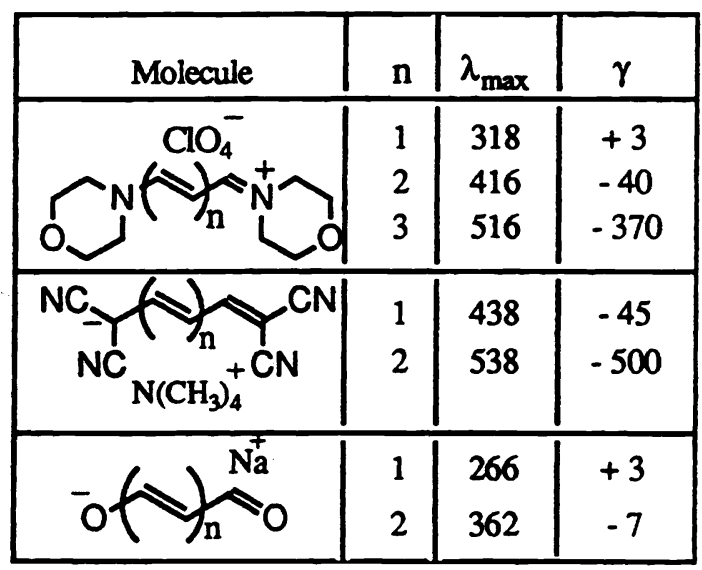

\section{SUMMARY}

Donor-acceptor polyenes of comparable conjugation length have been synthesized and their solvent-dependent hyperpolarizabilities probed by EFISH and THG measurements at $1907 \mathrm{~nm}$. Optimization in both a positive and negative sense, as well as sign changes in $\mu \cdot \beta$ and $\gamma$ were observed. These observations were explained by molecular structure changes (probed by $x$-ray crystallography, ${ }^{1}$ H-NMR, electronic absorption and Raman spectroscopies) resulting from the variation of mixing of neutral and charge-separated resonance forms upon changing the donor/acceptor strengths and solvent polarity. The trend of the geometry-dependent hyperpolarizabilities is fully consistent with the trends predicted using geometry-optimized electric-fielddependent AM1 and INDO-CI calculations and with the prediction that the polarizabilities are related by a derivative relationship. A separate series of ionic molecules possessing zero bond length alternation were found to exhibit negative third-order polarizabilities, also in agreement with the proposed structure-property relationships.

\section{ACKNOWLEDGMENTS}

The research described in this paper was performed in part by the Jet Propulsion Laboratory (JPL), California Institute of Technology, as part of its Center for Space Microelectronics Technology and was supported by the Advanced Research Projects Agency (administered by the Air Force Office of Scientific Research (AFOSR)) and the Ballistic Missiles Defense Initiative Organization, Innovative Science and Technology Office, through a contract with the National Aeronautics and Space Administration (NASA). We thank the AFOSR for support through grant \# F49620-92-J-0177 and AASERT for support through grant \# F49620-92-J-0278. Support at the Beckman Institute from the National Science Foundation (grant \# CHE9106689) and the North Atlantic Treaty Organization is gratefully acknowledged. G. B. thanks the National Research Council and NASA for a Resident Research Associateship at JPL. CBG thanks the JPL director's office for a postdoctoral fellowship. The authors would like to thank Professor Jean-Luc Brédas, Dr. Brian M. Pierce and Fabienne Meyers for helpful discussions and Howard D. Jones for expert technical assistance. 


\section{REFERENCES}

1. S. R. Marder, D. N. Beratan and L.-T. Cheng, Science, 252, pp. 103-106, 1991.

2. C. B. Gorman and S. R. Marder, Proc. Natl. Acad. Sci. USA, 90, pp. 11297-11301, 1993.

3. S. R. Marder, J. W. Perry, B. G. Tiemann, C. B. Gorman, S. Gilmour, S. Biddle and G. Bourhill, J. Am. Chem. Soc., 115, pp. 2524-2526, 1993.

4. P. Groth, Acta. Chem. Scand. B., 41, pp. 547-550, 1987.

5. S. R. Marder, B. G. Tiemann and W. P. Schaeffer, Unpublished observations.

6. L. G. S. Brooker, G. H. Keyes, R. H. Sprague, R. H. VanDyke, E. VanLare, G. VanZandt, F. L. White, H. W. J. Cressman and S. G. Dent, J. Am. Chem. Soc., 73, pp. 5332-5350, 1951.

7. S. R. Marder, J. W. Perry, G. Bourhill, C. B. Gorman, B. G. Tiemann and K. Mansour, Science, 261, pp. 186-189, 1993.

8. F. Meyers, S. R. Marder, B. M. Pierce and J.-L. Brédas, Submitted to Phys. Rev. Lett., 1994.

9. J. L. Oudar and D. S. Chemla, J. Chem. Phys., 66(6), pp. 2664-2668, 1977.

10. G. Bourhill, J.-L. Brédas, L.-T. Cheng, S. R. Marder, F. Meyers, J. W. Perry and B. G. Tiemann, Accepted for publication in J. Am. Chem. Soc., 1994.

11. A. F. Garito, J. R. Heflin, K. Y. Yong and O. Zamani-khamiri, Proc. SPIE-Int. Soc. Opt. Eng., 971, pp. 2-10, 1988.

12. B. M. Pierce, Proc. SPIE-Int. Soc. Opt. Eng., 1560, pp. 148-161, 1991.

13. C. W. Dirk, L.-T. Cheng and M. G. Kuzyk, Int. J. Quant. Chem., 43(1), pp. 27-36, 1992.

14. D. Lu, G. Chen, W. A. Goddard and J. W. Perry, to be published.

15. R. H. Baughman, B. E. Kohler, I. J. Levy and C. Spangler, Synth. Met., 11, pp. 37-52, 1985.

16. C. Reichardt, Solvent and Solvent Effects in Organic Chemistry, 2nd Edition, VCH, Weinheim, 1988.

17. L.-T. Cheng, W. Tam, S. H. Stevenson, G. R. Rikken and S. R. Marder, J. Phys. Chem., 95, pp. 10631-10643, 1991.

18. G. Bourhill, K. Mansour, B. G. Tiemann, C. B. Gorman, S. Biddle, S. R. Marder and J. W. Perry, Proc. SPIE-Int. Soc. Opt. Eng., 1853, pp. 208-220, 1993 and references therein.

19. G. Puccetti, M. Blanchard-Desce, I. Ledoux, J.-M. Lehn and J. Zyss, J. Phys. Chem., 97(37), pp. 9385-9391, 1993. 